\title{
Cytotoxicity and Collagen Expression Effects of Tideglusib Administration on Human Periodontal Cells: An In-Vitro Study
}

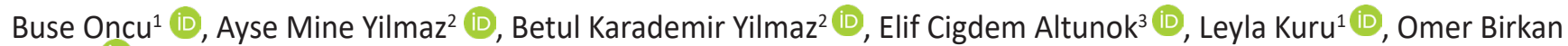 \\ Agrali ${ }^{1}$ \\ ${ }^{1}$ Marmara University, Faculty of Dentistry, Department of Periodontology, Istanbul, Turkey. \\ ${ }^{2}$ Marmara University, Faculty of Medicine/Genetic and Metabolic Diseases Research and Investigation Center, Department of Biochemistry, Istanbul, Turkey. \\ ${ }^{3}$ Yeditepe University, Faculty of Medicine, Department of Biostatistics and Medical Informatics, Istanbul, Turkey.
}

Correspondence Author: Omer Birkan Agrali

E-mail: omer.agrali@marmara.edu.tr

Received: $27.03 .2020 \quad$ Accepted: 20.04 .2020

\begin{abstract}
Objective: Tideglusib is a GSK-3 inhibitor activating Wnt/ $\beta$-catenin signaling pathway which has significant importance in regenerative response. The aim of this study was to evaluate the cytotoxicity and protein expression impacts of Tideglusib on human periodontal cell lines.

Methods: Cytotoxicity effect of different concentrations (50nM, 100nM, 200nM) of Tideglusib application on human gingival fibroblast (hGF), periodontal ligament fibroblast (hPDLF), and osteoblast (hOB) cell lines was determined. Type-I and III collagen expressions were evaluated after 24-hour application of 50nM Tideglusib.

Results: The cytotoxicity of 200nM Tideglusib was higher in hGF and hOB $(p<0.05)$, but no difference was found in hPDLF compared to the respective control group $(p>0.05)$. The hGF and hOB treated with 50nM Tideglusib expressed an increased level of Type-I collagen ( $p<0.05)$, but no difference was detected in the hPDLF compared to the respective control $(p>0.05)$. Type-III collagen expressions were similar between the test and control groups for each cell line ( $p>0.05)$.

Conclusion: Tideglusib is not cytotoxic at 50nM and 100nM concentrations and may have positive effect on bone regeneration rather than periodontal regeneration since it stimulated Type-I collagen production in hGF and hOB cells, but not in hPDLF.
\end{abstract}

Keywords: Cell biology, cell signaling biomolecules, Osteoblast(s), Tideglusib, Wnt/ $\beta$-catenin signaling pathway.

\section{INTRODUCTION}

Periodontal diseases are inflammatory diseases leading to progressive loss of tooth supporting structures (1). Conventional periodontal therapies utilized for the treatment of advanced periodontal diseases usually result in the formation of long junctional epithelium with a quality of repair (2). However, nowadays a complete regeneration is aimed, and new alveolar bone, cementum and periodontal ligament lost by disease are attempted to be established to their original structure and function using regenerative periodontal procedures. Although several materials have been tested, so far, none of them succeeded to accomplish complete regeneration (3).

Reformative responses of an organism include various signaling pathway activation cascades both in embryonic and adulthood periods. The activation of Wnt signaling pathway (WSP), one of the marked trails in the organisms, represents an early response to tissue damage and is required for the stimulation of cellular-based repair in all tissues (4). Wnt signaling mechanism can be in either active or inactive state. Following binding of the Wnt protein to cellular receptors, glycogen synthase kinase-3 (GSK-3) molecule which has great importance in regulating cell division cycle, is inhibited after a series of phosphorylation. Then, the transcription of the target genes of WSP begins. This stimulation plays a crucial role in proliferation, cell cycle and differentiation activities of the cells. Transcription of the target genes of signaling pathway is suppressed when Wnt does not bind to the cellular receptors (5). Several studies have shown that reduction in Wnt signaling causes bone loss and reduces regenerative capacity $(6,7)$. If the Wnt signaling is inhibited in the skeleton after fracture, due to the decline in the proliferation and earlier differentiation of skeletal stem and progenitor cells, non-fused bone appears (8). WSP activation leads to the proliferation of osteoblasts and fibroblasts resulting in the improvement of the parameters related to bone growth (9). In mammals, the healing of skin injuries usually ends with 
scar tissue, but when the WSP is activated, a fully functional epidermis can be achieved (10). Systemic use of Wnt agonist $\mathrm{R}$-spondin has been shown to induce mucosal regeneration in oral mucositis seen after chemotherapy (11). Irregular anatomical formations were observed after the inhibition of WSP in planarians possessing a high regenerative capacity (12). Similarly, healthy fin regeneration was impaired due to the inhibition of Wnt signaling in zebrafish (13). Moreover, inhibition of WSPs in animals having continuous retinal regeneration capacity results in interruption of their capability (14).

Current knowledge about the role of WSP on the development and maintenance of the periodontium is limited (1518). It was observed that root development discontinued in Wnt signaling inactivated mice (18). WSP-modulated mesenchymal mice odontoblast and cementoblast cells by persistent stabilization of ß-catenin have been shown to lead excessive dentin and cementum formation, and this finding was interpreted as Wnt signaling cascade might have therapeutic regenerative potential $(15,17,19)$. However, Nemoto et al. (20) established that the proliferation of cementoblast cells was supported by WSP activation. Furthermore, activation of the WSP has been reported to play an active role in cementoblast differentiation and cementum regeneration in rat periodontal defect model, and in in-vitro studies of human periodontal ligament cells $(9,17$, 21). In another study, it has been shown that lithium ions, known as activators of the WSP, increase the proliferation and differentiation of periodontal ligament cells after their release from bioactive scaffolds (9). Rooker et al. (17) found that the number of cells responding to the WSP was higher in the regions with increased proliferation of periodontal ligament cells. It is well-known that Wnt signaling plays a role in both osteoblast differentiation and proliferation, as well (22). A noteworthy increase in bone growth was observed in Wnt signaling activated mice (23). Popelut et al. (24) demonstrated that WSP activation might have a positive effect on implant osseointegration by enhancement of osteoblast activity, inhibition of osteoclast activity or differentiation of pluripotent stem cells.

Pharmacological inhibitors of GSK-3 are thought to be helpful in several areas such as Alzheimer's disease, some neurodegenerative diseases, Type-2 diabetes mellitus, cancer, psychiatric diseases and regenerative medicine (25). In an animal experimental periodontitis study, intraperitoneal administration of GSK-3 inhibitor was found to inhibit bone loss, suppress systemic cytokine response, local neutrophil infiltration and IL-17 expression (26). So far, many GSK-3 inhibitors including lithium, arylindolemaleimides, amino thiazoles, halomethylketone derivatives and thiadiazolidinones have been developed and shown to be applicable (26). Tideglusib belongs to the thiadiazolidinones group drug which acts as a non-ATP competitive GSK-3 inhibitor assessed in phase-Il clinical trials on a small-scale of subjects with Alzheimer disease since 2015. In a recent study, interestingly, Tideglusib was found to support the process of natural and functional reparative dentin formation in mice teeth with deep decays (4).

Under the light of these evidences, it was hypothesized that periodontal regeneration might be obtained by the use of Tideglusib to activate the WSP. Therefore, the aim of this study was to evaluate the effects of Tideglusib on human gingival fibroblast (hGF), periodontal ligament fibroblast (hPDLF), and osteoblast ( $\mathrm{hOB}$ ) cell lines pointing to assess the potential efficacy of Tideglusib in periodontal regeneration by measuring the cytotoxicity and proliferation levels together with Type-I and III collagen expression in these cells.

\section{METHODS}

\subsection{Study Plan}

hGF (Catalog no: CRL-2014, ATCC ${ }^{\circ}$, USA), hPDLF (Catalog no: CC-7049, Lonza, Switzerland) and hOB (Catalog no: CRL11372, ATCC ${ }^{\circ}$, USA.) cell lines were used to investigate the effects of Tideglusib administration. In the first stage of the study, MultiTox-Fluor Multiplex Cytotoxicity Test (Catalog no: G9200, Promega, USA) was applied to examine the effect of different Tideglusib concentrations on proliferation and cytotoxicity of the cells at the end of 24 hours. In the second stage, a common non-toxic dose of Tideglusib was determined and applied to all cell lines for 24 hours to investigate Type-I and Type-III collagen expressions evaluated by Western blot analysis. In accordance with this study plan, the cells treated with Tideglusib were designated as the test group, while the cells without Tideglusib served as the negative control group.

\subsection{Cell Culture}

Cells stored at $-152^{\circ} \mathrm{C}$ were allowed to thaw rapidly and centrifuged in $10 \mathrm{ml}$ medium at $1300 \mathrm{rpm}$ for 5 minutes. After the supernatant was removed, the pellet was resuspended and plated into media containing flasks. hGF cells were cultured in Dulbecco's Modified Eagle Medium (DMEM) (Catalog no: SLM-241-B, Sigma-Aldrich, Germany) supplemented with $10 \%$ fetal bovine serum (FBS) (Catalog no: 16000-036, GibcoTM, Thermo Fisher Scientific, USA), $2 \mathrm{mM}$ sodium pyruvate, 1\% L-glutamine (Catalog no: W368401, Sigma-Aldrich, Germany), high glucose, sodium bicarbonate and $0.1 \%$ amphotericin/gentamycin. Also, $0.1 \%$ fibroblast growth factor and $0.1 \%$ insulin-like growth factor were added to the culture medium for hPDLF cells. hOB cells were cultured in DMEM (Catalog no: P04-01549, Pan-Biotech, Germany) and DMEM/F12 (Catalog no: P0441500, Pan BioTech, Germany) medium containing medium appended with $10 \% \mathrm{FBS}, 1 \% \mathrm{~L}$-glutamine $(500 \mu \mathrm{l})$ and $0.1 \%$ amphotericin/gentamicin.

Cells were incubated in medium in a humid environment with $5 \% \mathrm{CO} 2$ at $37^{\circ} \mathrm{C}$, and cell proliferation was monitored for 24 hours. Cell passages with a confluence of $70 \%$ in the flasks were washed three times with phosphate-buffered saline (PBS) (Catalog no: P5493, Sigma-Aldrich, Germany) 
and incubated with trypsin-EDTA (Catalog no: P10-0235SP, Pan-Biotech, Germany) at $37^{\circ} \mathrm{C}$ for 5 minutes. Following the end of the trypsinization, cells were centrifuged at $1300 \mathrm{rpm}$ for 5 minutes and resuspended with the medium according to the pellet amount. In order to determine the cell count, $10 \mu$ l cell suspension was mixed with $10 \mu$ trypan blue. Cells were counted at $10 \mathrm{X}$ magnification in the light microscope (ZEISS Primovert, Germany). The cells stained with trypan blue were regarded as dead, whereas, the non-staineds as alive. The number of cells per $\mathrm{ml}$ was calculated according to the following formula (Cell count/ml=Mean count value $X$ dilution factor $\times 10^{4} \times 2$ ).

\subsection{Viability and Cytotoxicity Assays}

MultiTox-Fluor Multiplex Cytotoxicity Test was used to investigate Tideglusib's biocompatibility and was repeated three times for each cell Type. Two protease activities were measured in order to determine the cell viability and cytotoxicity in this experiment. For the determination of cell viability, $100 \mu \mathrm{l}$ of cell suspension containing 5000 cells was added to each well and incubated for 24 hours. After incubation, 50 nM, 100 nM and 200 nM Tideglusib (Catalog no: SML0339-50MG, Sigma-Aldrich, Germany) dissolved in dimethyl sulfoxide was applied to the test groups while the cells without Tideglusib served as negative control groups. At the end of 24 hours, $50 \mu$ l of GF-AFC reagent was added to all wells for the viability test. Plates were orbitally mixed for 5 minutes to ensure homogeneity and incubated at $37^{\circ} \mathrm{C}$ for 60 minutes. The plates were wrapped in aluminium foil and protected against light. The fluorescence was measured by the Multilabel Microplate Reader (EnSpire 2300 Multilabel Microplate Reader, Perkin Elmer, USA) with fluorescence of $\sim 400 \mathrm{nmEx} / \sim 505 \mathrm{~nm}$ wavelength.

For the cytotoxicity test, $50 \mu \mathrm{l} \mathrm{AAF-Glo}{ }^{\text {TM }}$ reagent was added to all wells and incubated in the dark at room temperature for 15 minutes. The absorbance values read in the Multilabel Microplate Reader were used for determining viability and cytotoxicity scores and specified as percentages of viability and cytotoxicity.

\subsection{Western Blotting}

Protein expressions of periodontal cells were examined by Western blotting method 24 hours after administration of $50 \mathrm{nM}$ Tideglusib, which was selected as the non-cytotoxic and most viability supporting dose. After incubation, the medium was removed, the cells were washed with PBS followed by cell lysis buffer administration containing 20
mM Tris- $\mathrm{HCl} \mathrm{pH}$ 7.5, $150 \mathrm{mM} \mathrm{NaCl}, 1 \mathrm{mM}$ Na2EDTA, $1 \mathrm{mM}$ EGTA, 1\% Triton, $2.5 \mathrm{mM}$ sodium pyrophosphate, $1 \mathrm{mM}$ beta glycerophosphate, $1 \mathrm{mM}$ Na3VO4, $1 \mu \mathrm{g} / \mathrm{ml}$ leupeptin and $1 \mathrm{mM}$ PMSF. Total protein extracts were separated by SDS-polyacrylamide gel electrophoresis and transferred to nitrocellulose membranes. Blots were blocked with $5 \%$ milk powder-tris buffered saline with tween 20 for 1 hour and incubated with polyclonal antibodies against Type-I collagen (Collagen I alpha 1 Antibody, Catalog no: 84336S, Cell Signaling Technology, USA) and Type-III collagen (Collagen III alpha 1 Antibody, Catalog no: NBP2-3332, Novus Biologicals, USA). The membrane was incubated with chemiluminescence kit (Western blotting luminol reagent, Santa Cruz Biotechnology, USA) in dark medium for 1 minute. The resulting radiation (ChemiDoc ${ }^{\mathrm{TM}}$ MP, Bio-Rad, USA) was displayed, and the density of the protein bands was calculated. The experiment was performed with four replicates for all cell types.

\subsection{Statistical Analysis}

The data were analyzed by using the Statistical Package for Social Sciences package program (SPSS for Windows, Release 25.0, IBM Inc., USA). Descriptive statistics are shown as mean \pm standard deviation, median, minimum and maximum. Data distribution was evaluated with Kolmogorov-Smirnov test. Kruskal-Wallis test was used to compare the variables that did not show normal distribution. Mann-Whitney $U$ test was used for paired comparisons. The results were interpreted with Bonferroni correction. Statistical significance was set as $\mathrm{p}<0.05$ level.

\section{RESULTS}

\subsection{The Effect of Tideglusib Administration on Cellular Viability and Cytotoxicity}

Table 1 shows the viability results of different concentrations of Tideglusib administration in all cell groups for 24 hours. Viability result of Tideglusib on hGF cells revealed no significant difference among the control and test groups (Table 1). On the other hand, there was a significant difference among the control and different concentrations of Tideglusib administrations in terms of viability effect on hPdlf cells ( $p=0.022$ ) (Table 1). 200 nM Tideglusib decreased the viability significantly compared to the $50 \mathrm{nM}$ Tideglusib application to hPDLF cells ( $p=0.037$ ) (Table 1$)$. The viability of hOB cells showed no significant difference for all administered concentrations (Table 1). 
Table 1. Comparison of viability among different concentrations of Tideglusib administration in all cell groups.

\begin{tabular}{|c|c|c|c|c|c|c|}
\hline & & \multicolumn{5}{|c|}{ Tideglusib Concentration } \\
\hline \multicolumn{2}{|c|}{ w } & $0 \mathrm{nM}$ & $50 \mathrm{nM}$ & $100 \mathrm{nM}$ & $200 \mathrm{nM}$ & $p^{*}$ \\
\hline \multirow{5}{*}{ 崩 す } & $\mathbf{N}$ & 3 & 3 & 3 & 3 & \multirow{5}{*}{0.639} \\
\hline & Mean \pm SD & $100.00 \pm 0.00$ & $134.60 \pm 61.63$ & $130.13 \pm 60.20$ & $124.08 \pm 63.64$ & \\
\hline & Median & 100.00 & 100.86 & 100.24 & 91.33 & \\
\hline & Minimum & 100.00 & 97.20 & 90.73 & 83.49 & \\
\hline & Maximum & 100.00 & 205.74 & 199.44 & 197.44 & \\
\hline \multirow{5}{*}{ 岂 } & $\mathbf{N}$ & 3 & 3 & 3 & 3 & \multirow{5}{*}{0.022} \\
\hline & Mean $\pm S D$ & $100.00 \pm 0.00$ & $107.51 \pm 6.23$ & $105.02 \pm 2.36$ & $97.63 \pm 1.68^{+}$ & \\
\hline & Median & 100.00 & 110.93 & 105.11 & 97.63 & \\
\hline & Minimum & 100.00 & 100.32 & 102.62 & 95.95 & \\
\hline & Maximum & 100.00 & 111.30 & 107.35 & 99.32 & \\
\hline \multirow{5}{*}{ 오 ᄚ } & $\mathbf{N}$ & 3 & 3 & 3 & 3 & \multirow{5}{*}{0.264} \\
\hline & Mean $\pm S D$ & $100.00 \pm 0.00$ & $115.77 \pm 3.20$ & $110.28 \pm 25.34$ & $101.98 \pm 8.90$ & \\
\hline & Median & 100.00 & 114.16 & 123.81 & 103.10 & \\
\hline & Minimum & 100.00 & 113.69 & 81.04 & 92.57 & \\
\hline & Maximum & 100.00 & 119.46 & 126.00 & 110.27 & \\
\hline
\end{tabular}

SD: Standard deviation, ${ }^{*}$ Kruskal Wallis test, $p<0.05,{ }^{+}$Different from $50 \mathrm{nM}$ Tideglusib administration, Mann Whitney-U Test with post-hoc Bonferroni correction, $p=0.037$

Table 2 shows the comparison of cytotoxicity values of all cell lines after 24-hour administration of Tideglusib at 50 nM, 100 $\mathrm{nM}$ and $200 \mathrm{nM}$ concentrations. Comparisons of the test and control groups in each cell line revealed significant cytotoxicity effect of Tideglusib in hGF ( $p=0.015), \operatorname{hPDLF}(p=0.023)$ and hOB cells $(p=0.015)$ (Table 2$)$. However, paired comparisons demonstrated no significant differences between the test and control groups in hPDLF when post-hoc Bonferroni correction was applied ( $p>0.05)$. In the paired comparisons, the cytotoxic effects of $200 \mathrm{nM}$ Tideglusib in the hGF and hOB cells were significantly higher compared to respective control groups ( $p=0.012, p=0.012$, respectively) (Table 2 ).

\subsection{The Effect of Tideglusib Administration on Type-I And Type-III Collagen Expression}

Following administration of $50 \mathrm{nM}$ Tideglusib onto the hGF cells, Type-I collagen expression in the test group was significantly higher than the control group $(p=0.029)$ (Figure 1) (Table 3). There was no significant difference between the test and control groups in terms of Type-I collagen expression in the hPdlf cells ( $p>0.05$ ) (Figure 1) (Table 3), Type-I collagen expression increased significantly in the test group compared to the control in the hOB cells $(p=0.029)$. (Figure 1), (Table 3). 
Table 2. Comparison of cytotoxicity among different concentrations of Tideglusib administration in all cell groups.

\begin{tabular}{|c|c|c|c|c|c|c|}
\hline \multicolumn{7}{|c|}{ Tideglusib Concentration (nM) } \\
\hline & & $0 \mathrm{nM}$ & $50 \mathrm{nM}$ & $100 \mathrm{nM}$ & $200 \mathrm{nM}$ & $\mathrm{p}^{*}$ \\
\hline \multirow{5}{*}{ 峁 § } & $\mathrm{N}$ & 3 & 3 & 3 & 3 & \multirow{5}{*}{0.015} \\
\hline & Mean $\pm S D$ & $0.00 \pm 0.00$ & $1780.30 \pm 203.33$ & $2342.23 \pm 82.75$ & $3329.18 \pm 564.40^{+}$ & \\
\hline & Median & 0.00 & 1816.17 & 2306.95 & 3290.34 & \\
\hline & Minimum & 0.00 & 1561.43 & 2282.97 & 2785.20 & \\
\hline & Maximum & 0.00 & 1963.32 & 2436.78 & 3912.0 & \\
\hline \multirow{5}{*}{ 㟒 } & $\mathbf{N}$ & 3 & 3 & 3 & 3 & \multirow{5}{*}{0.023} \\
\hline & Mean $\pm S D$ & $0.00 \pm 0.00$ & $1671.58 \pm 444.85$ & $5029.05 \pm 3326.79$ & $4782.91 \pm 2640.26$ & \\
\hline & Median & 0.00 & 1924.64 & 4350.70 & 4393.20 & \\
\hline & Minimum & 0.00 & 1157.93 & 2093.71 & 2359.18 & \\
\hline & Maximum & 0.00 & 1932.18 & 8642.74 & 7596.38 & \\
\hline \multirow{5}{*}{ 오 ஃ } & N & 3 & 3 & 3 & 3 & \multirow{5}{*}{0.015} \\
\hline & Mean $\pm S D$ & $0.00 \pm 0.00$ & $157.86 \pm 48.83$ & $265.88 \pm 25.64$ & $378.85 \pm 131.29^{+}$ & \\
\hline & Median & 0.00 & 130.30 & 254.40 & 310.56 & \\
\hline & Minimum & 0.00 & 129.05 & 247.99 & 295.79 & \\
\hline & Maximum & 0.00 & 214.25 & 295.26 & 530.22 & \\
\hline
\end{tabular}

SD: Standart deviation, *Kruskal Wallis Test, $p<0.05,{ }^{\dagger}$ Different from the control group, Mann Whitney-U Test with post-hoc Bonferroni correction, $p=0.012$.

Table 3. Type-I collagen expression in all cell groups after $50 \mathrm{nM}$ Tideglusib administration.

\begin{tabular}{|c|c|c|c|c|}
\hline \multicolumn{2}{|c|}{$\begin{array}{l}\text { Tideglusib } \\
\text { (nM) }\end{array}$} & $\begin{array}{c}\text { hGF } \\
\text { (Type-I Collagen/GAPDH) }\end{array}$ & $\begin{array}{c}\text { hPDLF } \\
\text { (Type-I Collagen/GAPDH) }\end{array}$ & $\begin{array}{c}\text { hOB } \\
\text { (Type-/ Collagen/GAPDH) }\end{array}$ \\
\hline \multirow{5}{*}{ 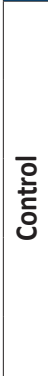 } & $N$ & 4 & 4 & 4 \\
\hline & Mean $\pm S D$ & $1.57 \pm 0.09$ & $0.11 \pm 0.03$ & $0.06 \pm 0.02$ \\
\hline & Median & 1.56 & 0.12 & 0.06 \\
\hline & Minimum & 1.47 & 0.07 & 0.03 \\
\hline & Maximum & 1.70 & 0.14 & 0.09 \\
\hline \multirow{5}{*}{$\begin{array}{l}\sum_{c} \\
\text { in }\end{array}$} & $N$ & 4 & 4 & 4 \\
\hline & Mean $\pm S D$ & $2.43 \pm 0.30$ & $0.19 \pm 0.12$ & $0.16 \pm 0.06$ \\
\hline & Median & 2.55 & 0.15 & 0.15 \\
\hline & Minimum & 1.98 & 0.10 & 0.09 \\
\hline & Maximum & 2.66 & 0.39 & 0.26 \\
\hline \multicolumn{2}{|l|}{$p^{*}$} & 0.029 & 0.343 & 0.029 \\
\hline
\end{tabular}

SD: Standard deviation. *Mann Whitney-U Test with post-hoc Bonferroni correction. $p<0.05$.

Type-III collagen expression analysis revealed no statistically significant difference between the test and control groups after 24 hours of $50 \mathrm{nM}$ Tideglusib administration onto each cell line ( $p>0.05$ ) (Figure 1) (Table 4). 


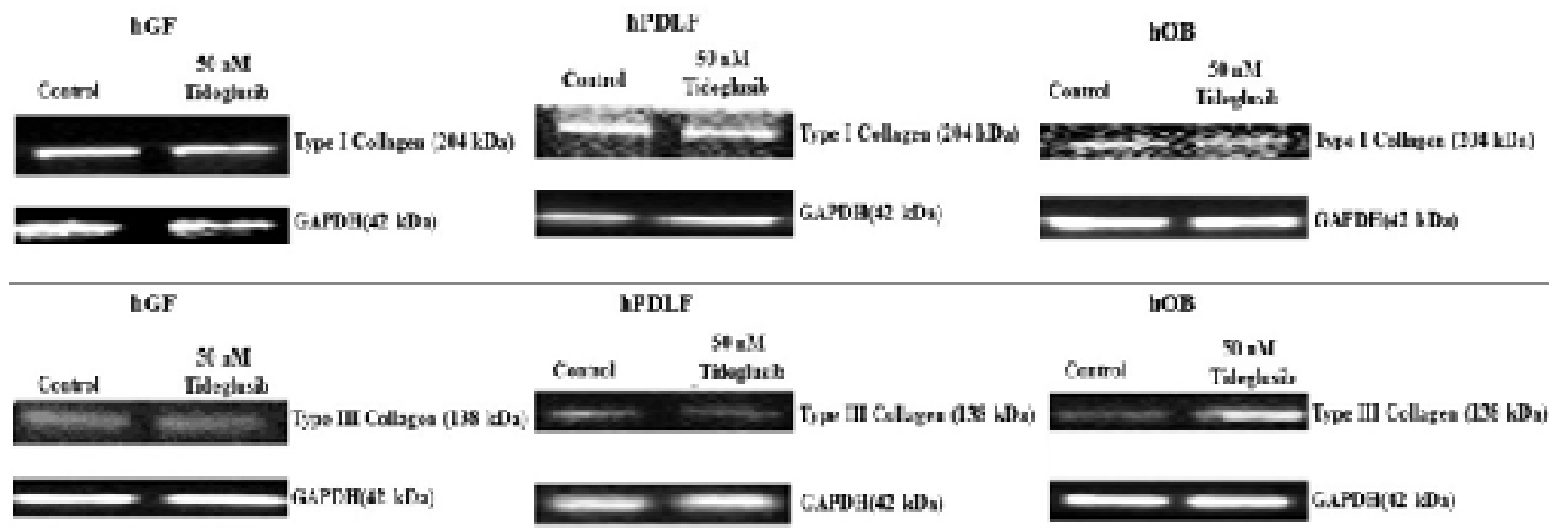

Figure 1. Representative Western blot images of all cell types. Immunoblotting for glyceraldehyde-3-phosphate dehydrogenase (GAPDH) (bottom panel) showed equal loading of the proteins in each lane. Bands were normalized to glyceraldehyde-3-phosphate dehydrogenase by densitometry.

Table 4. Type-III collagen expression in all cell groups after $50 \mathrm{nM}$ Tideglusib administration.

\begin{tabular}{|c|c|c|c|c|}
\hline \multicolumn{2}{|c|}{$\begin{array}{l}\text { Tideglusib } \\
\text { (nM) }\end{array}$} & $\begin{array}{c}\text { hGF } \\
\text { (Type-III Collagen/GAPDH) }\end{array}$ & $\begin{array}{c}\text { hPDLF } \\
\text { (Type-III Collagen/GAPDH) }\end{array}$ & $\begin{array}{c}\text { hOB } \\
\text { (Type-III Collagen/GAPDH) }\end{array}$ \\
\hline \multirow{5}{*}{$\begin{array}{l}\overline{0} \\
\text { 훙 }\end{array}$} & $N$ & 4 & 4 & 4 \\
\hline & Mean $\pm S D$ & $0.03 \pm 0.01$ & $0.24 \pm 0.07$ & $0.81 \pm 0.32$ \\
\hline & Median & 0.03 & 0.25 & 0.73 \\
\hline & Minimum & 0.02 & 0.15 & 0.54 \\
\hline & Maximum & 0.05 & 0.33 & 1.24 \\
\hline \multirow{5}{*}{ 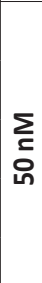 } & $\mathbf{N}$ & 4 & 4 & 4 \\
\hline & Mean \pm SD & $0.06 \pm 0.02$ & $0.18 \pm 0.09$ & $0.78 \pm 0.24$ \\
\hline & Median & 0.07 & 0.15 & 0.72 \\
\hline & Minimum & 0.03 & 0.12 & 0.57 \\
\hline & Maximum & 0.08 & 0.33 & 1.12 \\
\hline \multicolumn{2}{|c|}{ p* } & 0.20 & 0.34 & 1.00 \\
\hline
\end{tabular}

SD: Standard deviation. *Mann Whitney-U Test with post-hoc Bonferroni correction. $p<0.05$.

\section{DISCUSSION}

WSP activation is known as an early response to the natural repair mechanism against tissue damage in mammals (4). Therefore, the main target in repair/regeneration is WSP stimulation via several different conducts, one of which is GSK inhibition. Tideglusib is a small molecule GSK-3 inhibitor which is efficient in reparative dentin formation, osteoblast proliferation and tissue repair (4). In this study, the influence of Tideglusib on periodontal cells in terms of cytotoxicity, viability and Type-I and III collagen expressions were investigated in order to clarify any potential role of this molecule in periodontal regeneration. This is the first study evaluating the response of periodontal cells treated with Tideglusib.
MultiTox assay protocol was applied to investigate the cytotoxicity and proliferation outcomes of Tideglusib on human periodontal cell lines. These tests are performed by the separation of live and dead cells based on protease and esterase activity (27). Since protease substrates do not disrupt cell viability throughout the experimental period, this assay can be repeated several times, unlike other dye exclusion (27). It is also possible to measure the number of both live and dead cells at the same time. Although other luminescence-based cytotoxicity tests without causing cell destruction are also available in the fields of toxicology and pharmacology, the stability of the tests mentioned above is relatively low as well as the half-life of the luciferase signals is short (27). 
The periods in which cellular responses are intended to be evaluated in cell culture studies may vary according to the study plan. Osteoblast activity may be assessed either shortterm or long-term ranging from 15 minutes to 60 hours (2830 ), whereas 24-hour and 72-hour incubation periods were appraised in studies concerned with fibroblast activity (31). In this study, a 24-hour incubation period was performed as suggested by Kim et al. (32) and Scotchford et al. (33)

A recent study of Neves et al. (4) suggesting Tideglusib stimulated the renewal of living stem cells in tooth pulp inspired the concentrations used in our study. Different concentrations of Tideglusib (50 nM, 100 nM and 200 $\mathrm{nM}$ ) were used for cytotoxicity and proliferation assays in this study. Cytotoxicity increased significantly after 24 hours administration of $200 \mathrm{nM}$ Tideglusib in the hGF cells $(p=0.012)$. In the proliferation experiment, there was no significant increase in the test groups compared to the control group. Some studies in the literature demonstrated that WSP activation causes fibrosis by increasing fibroblast activation and proliferation $(34,35)$. It has been shown that Wnt activation can lead to increased proliferation of lung fibroblasts, increased differentiation of fibroblasts to myofibroblasts together with increased myofibroblast count (36). Hamburg et al. (37) reported that continuous activation of $\beta$-catenin, a cytosolic protein that has been associated with numerous biological tasks utilizing a transcriptional co-activator in the WSP, may cause fibrosis depending on its enhancing result in the number of dermal fibroblasts. In contrast to these studies, Wnt activation and GSK-3 inhibition have been shown to decrease gingival growth by decreasing TGF- $\beta 1$ expression in gingival fibroblasts (38). In our study for the first time, Tideglusib did not exert any proliferative effect on hGF cells.

The cytotoxicity and proliferation effects of Tideglusib on hPDLF cells were also evaluated in this study. Rooker et al. (17) found in their immunohistochemical study that the Wnt signaling response was higher in the proliferation areas in the periodontal ligament, however, so far no study exists in the literature investigating the impact of Tideglusib on hPDLF cells. Han et al. (9) showed that the proliferation of hPDLF cells increased compared to the control group when lithium, as a WSP activator, was applied. In our study, no significant difference was found between the test groups and the control group $(p>0.05)$. While our result does not correlate with the previous studies $(9,17)$ investigating the influence of WSP activation on hPDLF cells using Wnt modulators such as lithium or Dickkopf molecules, this is the first study to asses the effect of Tideglusib as a WSP activator, and therefore, it may be necessary to evaluate the impact of Tideglusib on hPDLF cells for more extended periods of time.

While Tideglusib application did not demonstrate any upshot on the proliferation of hOB cells, the cytotoxicity of hOB cells increased compared to the control group after the use of 200 $n M$ Tideglusib ( $p=0.012$ ). Morvan et al. (39) observed raised proliferative impression on the osteoblasts of WSP induced mice. Babij et al. (23) stated that when the WSP is activated, there will be an increase in bone mass since the number of osteoblasts enhanced. Caetano-Lopes et al. (40) reported that activation of the WSP is a factor in osteoblast differentiation and proliferation. Moreover, Westendorf et al. (22) and Lerner et al. (41) have shown that WSP plays a critical role in the trabecular and cortical bone mechanism, but so far there are no studies published on the effect of Tideglusib on osteoblast cells. In the present study, osteoblast proliferation was not affected by WSP activation initiated with Tideglusib, which is a new promising molecule with its intriguing theraupeutic efficiency. Further detailed and comprehensive studies are needed to clarify the consequence of WSP activation on osteoblast cells through different pathways.

Based on the findings of cytotoxicity and proliferation assays, the non-cytotoxic dose of Tideglusib that supports the viability was determined as $50 \mathrm{nM}$, which was further used for Type-I and Type-III collagen experiments. Type-I collagen is the main component of connective tissues such as gingiva, periodontal ligament, dentin, cementum and bone. Also, Type-I collagen is considered as a marker for osteoblast differentiation (42). Type-III collagen exists in the gingiva, periodontal ligament, cementum, skin, blood vessels, fetal tissues and plays an essential role in collagen production/destruction mechanism (43). Type-III collagen provides tissue resistance at the early stages of healing and leaves its place to Type-I collagen as the healing is complete (43). However, there is a limited number of studies evaluating protein expression associated with the WSP. Minear et al. (6) created a biochemical approach to increase the duration and strength of Wnt signaling at the sites of the skeletal wound and demonstrated that Type-I collagen appeared faster in the wound area of WSP activated mutant mice as a result of more vigorous proliferation and earlier differentiation of skeletal stem/progenitor cells. Xiang et al. (44) established a significant decrease in Type-I collagen expression from cardiac fibroblasts of mice having a genetic deficiency of $\beta$-catenin molecule. In the light of these studies, Type-I and Type-III collagens were chosen as common proteins for three cell lines to examine the potential regenerative capacity of Tideglusib in our research.

After administration of $50 \mathrm{nM}$ Tideglusib to the hGF cell line, Type-I collagen level was detected to increase significantly compared to the control group $(p=0.029)$. Roh et al. (45) hypothesized that the stabilization of $\beta$-catenin molecule which exhibits regulatory properties on target gene expression for regeneration/repair after its translocation to the cell nucleus and has a manner of central transducer property for the WSP in fibroblasts might have a positive influence on Type-I collagen synthesis. They reported higher Type-I collagen expression in keloid tissue fibroblasts, presenting elevated fibrotic activity compared to the normal dermal fibroblasts. Similarly, improved expression of Type-I collagen was observed in the liver fibroblasts of WSP activated mice $(46,47)$. Moreover, Bergmann et al. (34) demonstrated that after inhibition of GSK-3 aiming to activate WSP, a significant upsurge in Type-I collagen expression was displayed in cultured mice dermal fibroblasts. Svegliati et al. (48) confirmed an escalation in the amount of Type-I collagen 
expression in conjunction with WSP activation by fibroblasts causing fibrosis in the punch biopsies of systemic sclerosis patients. Our result that a rise in Type-I collagen level was observed in hGF cells was found to be compatible with the studies performed with other Types of fibroblast cells in the literature $(34,46-48)$.

On the contrary, hPDLF cells treated with Tideglusib expressed a similar level of Type-I collagen as the control cells $(p>0.05)$. This finding is not parallel to the previous studies showing that Wnt signaling activation stimulates Type-I collagen expression in dermal, cardiac and liver fibroblasts $(34,45,46)$. Nevertheless, the collagen expression in hPDLF cells treated with Tideglusib has not been investigated so far according to our knowledge. There is, however, a prosperity of data concerning the role of Wnt signaling in mediating the behaviours of other types of stem cells (17).

Besides mineralized nodule formation, expressions of Type-I collagen, alkaline phosphatase, osteonectin and Runx2 were evaluated as bone formation markers in studies investigating the result of WSP activation on osteoblast cells $(15,24,39)$. Type-I collagen exists abundantly both in the organic matrix of gingiva and bone tissues $(42,47)$. In our study, as a common marker for both gingiva and bone tissues, Type-I collagen level was measured after the administration of $50 \mathrm{nM}$ Tideglusib to the hOB cells and found significantly higher compared to the control group ( $p=0.029$ ). Glass et al. (49) observed a significant increase in the bone mass accompanied by higher Type-I collagen expression in osteoblast cells of Wnt signaling activated mice. Kim et al. (15) presented higher Type-I collagen release and accelerated osteoblast differentiation together with Wnt signaling activation. Popelut et al. (24) examined the consequence of Wnt signaling activation on implant osseointegration and observed a substantial rise in Type-I collagen level after signal path stimulation. Activation of WSPs provides an escalation in bone mass and increases osteoblast activity, and the data revealed from our study may support the findings that Tideglusib may play a role in the intensification of bone mass.

There is a limited number of studies in the literature exploring the significance of WSP activation on Type-III collagen expression $(45,50)$. In our study, Type-III collagen expression was not statistically different between the test and control groups for each cell line. Similarly, Roh et al. (45) did not observe any sense of $\beta$-catenin stabilization on TypeIII collagen release in dermal fibrosis tissue fibroblasts. In contrast to the findings of our study and Roh et al. (45), Ge et al. (50) reported Type-III collagen increase in hepatic tissue fibrosis and decreased hepatic cell proliferation after the blockage of Wnt signaling. However, the differences in the methods applied and cell lines may explain this inconsistency.

In our study, 24-hour incubation with Tideglusib did not cause any change in Type-I collagen expression in the hPDLF cell. Similarly, Tideglusib did not stimulate Type-III collagen expression at 24 hours in any cell line. Short evaluation period may be considered as a limitation of our study. Protein expressions were evaluated in the cell culture studies at periods ranging from 24 hours to 72 hours $(15,24,28,31)$. Although, Popelut et al. (24) did not observe an upturn in the amount of collagen after 24-hour protein release test, they found a significant upsurge after 48 hours of evaluation. Therefore, it was concluded that more prolonged periods of time are needed to examine the influence of Tideglusib on periodontal cells.

\section{CONCLUSION}

It can be concluded that tideglusib molecule is not cytotoxic at $50 \mathrm{nM}$ and $100 \mathrm{nM}$ concentrations and may have a possible positive effect on bone regeneration rather than periodontal regeneration since it causes type-I collagen increase in hGF and hOB cells, but not in hPDLF. However, our study is the first to investigate the impact of tideglusib on periodontal cells. Further studies are warranted to examine a wide range of healing markers to provide a clearer view for screening the power of Tideglusib on periodontal regeneration.

Acknowledgments: We thank our colleagues from Marmara University Genetic and Metabolic Diseases Research and Investigation Center who provided insight and expertise that greatly assisted the research.

\section{REFERENCES}

[1] Kocher T, Konig J, Dzierzon U, Sawaf H, Plagmann HC. Disease progression in periodontally treated and untreated patients-a retrospective study. J Clin Periodontol 2000; 27:866-872.

[2] Melcher AH. On the repair potential of periodontal tissues. J Periodontol 1976; 47:256-260.

[3] Bosshardt DD, Sculean A. Does periodontal tissue regeneration really work? Periodontol 2000 2009; 51:208-219.

[4] Neves VC, Babb R, Chandrasekaran D, Sharpe PT. Promotion of natural tooth repair by small molecule GSK3 antagonists. Scientific reports 2017; 7:39654.

[5] Willert K, Nusse R. beta-catenin: a key mediator of Wnt signaling. Curr Opin Genet Dev 1998; 8:95-102.

[6] Minear S, Leucht P, Jiang J, Liu B, Zeng A, Fuerer C, Nusse FR, Helms JA. Wnt proteins promote bone regeneration. Sci Transl Med 2010; 2:29ra30.

[7] Whyte JL, Smith AA, Helms JA. Wnt signaling and injury repair. Cold Spring Harbor perspectives in biology 2012; 4:a008078.

[8] Leucht P, Minear S, Ten Berge D, Nusse R, Helms JA. Translating insights from development into regenerative medicine: the function of Wnts in bone biology. Semin Cell Dev Biol 2008; 19:434-443.

[9] Han PP, Wu CT, Chang J, Xiao Y. The cementogenic differentiation of periodontal ligament cells via the activation of $\mathrm{Wnt} /$ betacatenin signalling pathway by Li+ ions released from bioactive scaffolds. Biomaterials 2012; 33:6370-6379.

[10] Ito M, Yang Z, Andl T, Cui C, Kim N, Millar SE, Cotsarelis G. Wntdependent de novo hair follicle regeneration in adult mouse skin after wounding. Nature 2007; 447:316-320.

[11] Zhao J, Kim KA, De Vera J, Palencia S, Wagle M, Abo A. R-Spondin1 protects mice from chemotherapy or radiationinduced oral mucositis through the canonical Wnt/betacatenin pathway. Proc Natl Acad Sci U S A 2009; 106:2331-2336. 
[12] Petersen CP, Reddien PW. A wound-induced Wnt expression program controls planarian regeneration polarity. Proc Natl Acad Sci U S A 2009; 106:17061-17066.

[13] Kawakami Y, Rodriguez Esteban C, Raya M, Kawakami H, Marti M, Dubova I, Belmonte JCl. Wnt/beta-catenin signaling regulates vertebrate limb regeneration. Genes Dev 2006; 20:3232-3237.

[14] Kubo F, Takeichi M, Nakagawa S. Wnt2b controls retinal cell differentiation at the ciliary marginal zone. Development 2003; 130:587-598.

[15] Kim JB, Leucht P, Lam K, Luppen C, Ten Berge D, Nusse R, Helms JA. Bone regeneration is regulated by wnt signaling. J Bone Miner Res 2007; 22:1913-1923.

[16] Lim WH, Liu B, Cheng D, Williams BO, Mah SJ, Helms JA. Wnt signaling regulates homeostasis of the periodontal ligament. J Periodontal Res 2014; 49:751-759.

[17] Rooker SM, Liu B, Helms JA. Role of Wnt signaling in the biology of the periodontium. Dev Dyn 2010; 239:140-147.

[18] Zhang R, Yang G, Wu X, Xie J, Yang X, Li T. Disruption of Wnt/ beta-catenin signaling in odontoblasts and cementoblasts arrests tooth root development in postnatal mouse teeth. Int J Biol Sci 2013; 9:228-236.

[19] Kim TH, Lee JY, Baek JA, Lee JC, Yang X, Taketo MM, Jiang R, Cho ES. Constitutive stabilization of ss-catenin in the dental mesenchyme leads to excessive dentin and cementum formation. Biochem Biophys Res Commun 2011; 412:549-555.

[20] Nemoto E, Koshikawa Y, Kanaya S, Tsuchiya M, Tamura M, Somerman MJ, Shimauchi H. Wnt signaling inhibits cementoblast differentiation and promotes proliferation. Bone 2009; 44:805-812.

[21] Han P, Ivanovski S, Crawford R, Xiao Y. Activation of the Canonical Wnt Signaling Pathway Induces Cementum Regeneration. J Bone Miner Res 2015; 30:1160-1174.

[22] Westendorf JJ, Kahler RA, Schroeder TM. Wnt signaling in osteoblasts and bone diseases. Gene 2004; 341:19-39.

[23] Babij P, Zhao W, Small C, Kharode Y, Yaworsky PJ, Bouxsein ML, Reddy SP, Bodine PVN, Robinson JA, Bhat BM, Marzolf J, Moran RA, Bex FJ. High bone mass in mice expressing a mutant LRP5 gene. J Bone Miner Res 2003; 18:960-974.

[24] Popelut A, Rooker SM, Leucht P, Medio M, Brunski JB, Helms JA. The acceleration of implant osseointegration by liposomal Wnt3a. Biomaterials 2010; 31:9173-9181.

[25] Meijer L, Flajolet M, Greengard P. Pharmacological inhibitors of glycogen synthase kinase 3. Trends Pharmacol Sci 2004; 25:471-480.

[26] Adamowicz K, Wang H, Jotwani R, Zeller I, Potempa J, Scott DA. Inhibition of GSK3 abolishes bacterial-induced periodontal bone loss in mice. Mol Med 2012; 18:1190-1196.

[27] Niles AL, Moravec RA, Eric Hesselberth P, Scurria MA, Daily WJ, Riss TL. A homogeneous assay to measure live and dead cells in the same sample by detecting different protease markers. Anal Biochem 2007; 366:197-206.

[28] Bowers KT, Keller JC, Randolph BA, Wick DG, Michaels CM. Optimization of surface micromorphology for enhanced osteoblast responses in vitro. Int J Oral Maxillofac Implants 1992; 7:302-310.

[29] Campoccia D, Arciola CR, Cervellati M, Maltarello MC, Montanaro L. In vitro behaviour of bone marrow-derived mesenchymal cells cultured on fluorohydroxyapatite-coated substrata with different roughness. Biomaterials 2003; 24:587596.
[30] Yliheikkila PK, Masuda T, Ambrose WW, Suggs CA, Felton DA, Cooper LF. Preliminary comparison of mineralizing multilayer cultures formed by primary fetal bovine mandibular osteoblasts grown on titanium, hydroxyapatite, and glass substrates. Int J Oral Maxillofac Implants 1996; 11:456-465.

[31] Kook SH, Lee D, Cho ES, Heo JS, Poudel SB, Ahn YH. Hwang JW, Ji H, Kim JG, Lee JC. Activation of canonical Wnt/beta-catenin signaling inhibits $\mathrm{H} 2 \mathrm{O} 2$-induced decreases in proliferation and differentiation of human periodontal ligament fibroblasts. Mol Cell Biochem 2016; 411:83-94.

[32] Kim HJ, Kim SH, Kim MS, Lee EJ, Oh HG, Oh WM, Park SW, Kim WJ, Lee GJ, Choi NG, Koh JT, Dinh DB, Hardin RR, Johnson K, Sylvia VL, Schmitz JP, Dean DD. Varying Ti-6Al-4V surface roughness induces different early morphologic and molecular responses in MG63 osteoblast-like cells. J Biomed Mater Res A 2005; 74:366-373.

[33] Scotchford CA, Ball M, Winkelmann M, Voros J, Csucs C, Brunette DM, Danuser G, Textor M. Chemically patterned, metal-oxide-based surfaces produced by photolithographic techniques for studying protein - and cell-interactions. II: Protein adsorption and early cell interactions. Biomaterials 2003; 24:1147-1158.

[34] Bergmann C, Akhmetshina A, Dees C, Palumbo K, Zerr P, Beyer C, Zweina J, Distler O, Schett G, Distler JHW. Inhibition of glycogen synthase kinase $3 \beta$ induces dermal fibrosis by activation of the canonical Wnt pathway. Annals of the rheumatic diseases 2011; 70:2191-2198.

[35] Burgy O, Königshoff M. The WNT signaling pathways in wound healing and fibrosis. Matrix Biology 2018.

[36] Akhmetshina A, Palumbo K, Dees C, Bergmann C, Venalis P, Zerr P, Horn A, Kireva T, Beyer C, Zwerina J, Schneider H, Sadowski A, Riener MO, MacDougald OA, Distler O, Schett G, Distler JHW. Activation of canonical Wnt signalling is required for TGF-beta-mediated fibrosis. Nat Commun 2012; 3:735.

[37] Hamburg EJ, Atit RP. Sustained beta-catenin activity in dermal fibroblasts is sufficient for skin fibrosis. J Invest Dermatol 2012; 132:2469-2472.

[38] Bahammam M, Black SA, Jr., Sume SS, Assaggaf MA, Faibish M, Trackman PC. Requirement for active glycogen synthase kinase3beta in TGF-beta1 upregulation of connective tissue growth factor (CCN2/CTGF) levels in human gingival fibroblasts. Am J Physiol Cell Physiol 2013; 305:C581-590.

[39] Morvan F, Boulukos K, Clement-Lacroix P, Roman Roman S, SucRoyer I, Vayssiere B, Ammann P, Martin P, Pinho S. Deletion of a single allele of the Dkk1 gene leads to an increase in bone formation and bone mass. J Bone Miner Res 2006; 21:934-945.

[40] Caetano-Lopes J, Canhao H, Fonseca JE. Osteoblasts and bone formation. Acta Reumatol Port 2007; 32:103-110.

[41] Lerner UH, Ohlsson C. The WNT system: background and its role in bone. J Intern Med 2015; 277:630-649.

[42] Dacic S, Kalajzic I, Visnjic D, Lichtler AC, Rowe DW. Col1a1driven transgenic markers of osteoblast lineage progression. J Bone Miner Res 2001; 16:1228-1236.

[43] Mariotti A. The extracellular matrix of the periodontium: dynamic and interactive tissues. Periodontol 2000 1993; 3:3963.

[44] Xiang FL, Fang M, Yutzey KE. Loss of beta-catenin in resident cardiac fibroblasts attenuates fibrosis induced by pressure overload in mice. Nat Commun 2017; 8:712.

[45] Roh MR, Kumar R, Rajadurai A, Njauw C, Ryoo UH, Chung $\mathrm{KY}$, Tsao H. Beta-catenin causes fibrotic changes in the 
extracellular matrix via upregulation of collagen I transcription. Br J Dermatol 2017; 177:312-315.

[46] Beljaars L, Daliri S, Dijkhuizen C, Poelstra K, Gosens R. WNT-5A regulates TGF $\beta$-related activities in liver fibrosis. American Journal of Physiology-Heart and Circulatory Physiology 2017.

[47] Beyer C, Schramm A, Akhmetshina A, Dees C, Kireva T, Gelse $\mathrm{K}$, Sonnylal S, Crombrugghe B, Taketo MM, Distler O, Schett $G$, Distler JHW. $\beta$-catenin is a central mediator of pro-fibrotic Wnt signaling in systemic sclerosis. Annals of the rheumatic diseases 2012; 71:761-767.

[48] Svegliati S, Marrone G, Pezone A, Spadoni T, Grieco A, Moroncini G, Grieco D, Vinciguerra $M$, Agnese $S$, Jungel $A$,
Distler O, Musti AM, Gabrielli A, Avvedimento EV. Oxidative DNA damage induces the ATM-mediated transcriptional suppression of the Wnt inhibitor WIF-1 in systemic sclerosis and fibrosis. Sci Signal 2014; 7:ra84.

[49] Glass DA, 2nd, Bialek P, Ahn JD, Starbuck M, Patel MS, Clevers $\mathrm{H}$, Taketo MM, Long F, McMahon AP, Lang RA, Karsenty G. Canonical Wnt signaling in differentiated osteoblasts controls osteoclast differentiation. Dev Cell 2005; 8:751-764.

[50] Ge W-S, Wang Y-J, Wu J-X, Fan J-G, Chen Y-W, Zhu L. $\beta$-catenin is overexpressed in hepatic fibrosis and blockage of Wnt/ $\beta$-catenin signaling inhibits hepatic stellate cell activation. Molecular medicine reports 2014; 9:2145-2151.

How to cite this article: Oncu B, Yilmaz AM, Karademir Yilmaz B, Altunok EC, Kuru L, Agrali OB. Cytotoxicity and Collagen Expression Effects of Tideglusib Administration on Human Periodontal Cells: An In-Vitro Study. Clin Exp Health Sci 2020; 10: 153-162. DOI: 10.33808/clinexphealthsci.709924 